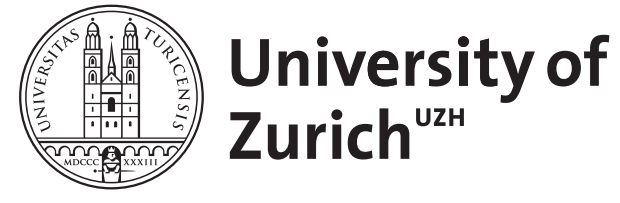

\title{
Neuromodulation of Urinary Tract Function
}

Kessler, Thomas M ; Birder, Lori A ; Gomery, Pablo

DOI: https://doi.org/10.1056/NEJMcibr1900051

Posted at the Zurich Open Repository and Archive, University of Zurich

ZORA URL: https://doi.org/10.5167/uzh-171352

Journal Article

Published Version

Originally published at:

Kessler, Thomas M; Birder, Lori A; Gomery, Pablo (2019). Neuromodulation of Urinary Tract Function. New England Journal of Medicine, 380(21):2067-2069.

DOI: https://doi.org/10.1056/NEJMcibr1900051 


\title{
C L I N I C A I M P L I A TIONS OF B A I C R E E A R C H
}

Elizabeth G. Phimister, Ph.D., Editor

\section{Neuromodulation of Urinary Tract Function}

\author{
Thomas M. Kessler, M.D., Lori A. Birder, Ph.D., and Pablo Gomery, M.D.
}

The function of the lower urinary tract - to store and periodically eliminate urine - seems simple, but its neuronal control is complex and not fully understood, and dysfunction is common. Functional electrical stimulation of the tibial, pudendal, and sacral nerves involves spinal cord reflexes and brain networks through peripheral afferents. These targets for both the inhibition and stimulation of lower urinary tract function have been potential therapeutic options in urology since the 1990s. Although the technology has progressively improved, restoration of lower urinary tract function has met with limited success. A study recently reported by Mickle et al. ${ }^{1}$ harnesses optogenetics to control function of the lower urinary tract.

Optogenetic approaches, which use lightresponsive proteins to selectively turn on or off specific cell populations, have completely changed the ability to modulate cellular activity in a number of targets. Although the science of neuronal control got under way in 1999 when Sir Francis Crick first hypothesized that light could be used to control the action potential of neurons, it took 6 years before Boyden and colleagues published their seminal work in $2005,{ }^{2}$ showing that they could stimulate neuronal activation with precise timing by transfecting genes encoding lightactivated opsins into specific neurons using a viral vector. The activated opsin, in turn, activates neuronal firing.

In 2017, Park and colleagues found that they could use opsins specifically to stimulate or inhibit contraction of smooth-muscle cells in the bladder. ${ }^{3}$ Depolarization of the cells with the use of one opsin (channelrhodopsin-2) activated vesical contraction, whereas hyperpolarization with another (halorhodopsin) inhibited contraction. ${ }^{3}$ Park et al. underscored that a singular advantage of the strategy is target specificity through genetic delivery (including use of a viral vector) of photosensitive membrane proteins into specific cells of the nervous system. Before their study, functional electrical neuromodulation could produce simultaneous contraction of both detrusor smooth muscle and striated muscle in the external urethral sphincter, which would not be desirable for lower urinary tract function.

In their recent study, Mickle et al. modified this approach by incorporating optogenetic methods to selectively modulate neurons and thereby affect bladder function in a rat model. They used a closed-loop wireless system with an implantable device wrapped around the bladder of freely moving rats (Fig. 1). The device measures changes in resistance during bladder filling and emptying. For example, when the bladder is overactive, a signal is sent to activate a small light-emitting diode (LED) which, in turn, activates a light-sensitive archaeal protein - an inhibitory opsin, archaerhodopsin-3 (Arch), that is expressed in specific neural targets - to selectively inhibit the emptying of the bladder, thereby restoring normal function. They induced inflammation in the otherwise healthy animals by intraperitoneal injection of cyclophosphamide to test the hypothesis that the activation of Arch would reduce neural activity and, by delivering a corrective signal, restore bladder storage function. The results supported the hypothesis: activation of Arch did indeed reduce neural activity and restored normal bladder storage function.

Nevertheless, several issues must be solved before optogenetic findings in studies involving animals can be translated to daily clinical practice for lower urinary tract control. The safety of the viral vector that is required for optogenetics in humans needs to be proved not only in the 


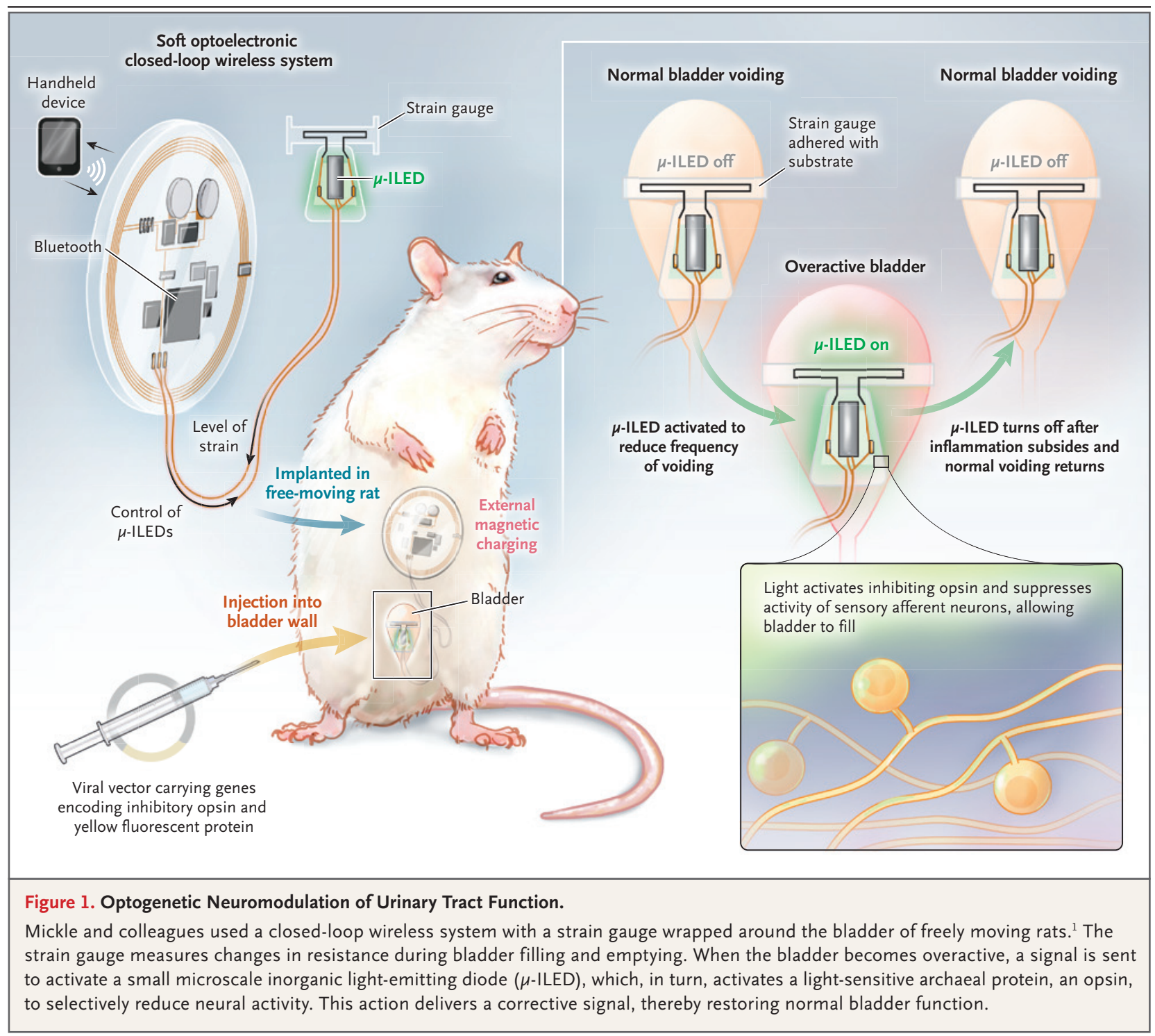

short term but also in the long term. This challenge remains a major one, especially considering that there are still no clinically implemented optogenetic applications in humans. In the model of Mickle et al., a stretch sensor wrapped around the bladder is the key element for monitoring lower urinary tract function. Reaction to the presence of a foreign body and a dwindling of bladder compliance may impede implementation of this system. Miniaturization of the module, enhancing it with a pressure sensor, and implanting it directly into the bladder wall - for instance, by injecting a combined mini-stretchpressure sensor with the use of cystoscopy (similar to intradetrusor injections of onabotulinumtoxinA) - seems worthy of exploration. The thicker bladder wall in humans would probably impede light transmission, as compared with that in rats, and so might warrant different light-delivery modes, such as direct placement inside the bladder. Red light may be necessary, since it penetrates biologic tissue much more efficiently than does light of other wavelengths.

Although there are other hurdles to overcome, the closed-loop optogenetic neuromodulation system developed by Mickle and colleagues opens new avenues to restore lower urinary tract function in humans. As a result of the closed-loop 
system, neuromodulation is only provided when the device detects a problem. And, in contrast to current functional electrical stimulation of the tibial, pudendal, and sacral nerves, optogenetic innervation can target specific neurons in the lower urinary tract and thus can avoid interfering with bowel or sexual function.

Disclosure forms provided by the authors are available with the full text of this article at NEJM.org.

From the Department of Neuro-Urology, Balgrist University Hospital, University of Zurich, Zurich, Switzerland (T.M.K.); the Departments of Medicine and Pharmacology and Chemical
Biology, University of Pittsburgh, Pittsburgh (L.A.B.); and the Department of Urology, Massachusetts General Hospital, Boston (P.G.).

1. Mickle AD, Won SM, Noh KN, et al. A wireless closed-loop system for optogenetic peripheral neuromodulation. Nature 2019;565:361-5.

2. Boyden ES, Zhang F, Bamberg E, Nagel G, Deisseroth K. Millisecond-timescale, genetically targeted optical control of neural activity. Nat Neurosci 2005;8:1263-8.

3. Park JH, Hong JK, Jang JY, et al. Optogenetic modulation of urinary bladder contraction for lower urinary tract dysfunction. Sci Rep 2017;7:40872.

DOI: 10.1056/NEJMcibr1900051

Copyright (c) 2019 Massachusetts Medical Society.

APPLY FOR JOBS AT THE NEJM CAREERCENTER

Physicians registered at the NEJM CareerCenter can apply for jobs electronically. A personal account created when you register allows you to apply for positions, using your own cover letter and CV, and keep track of your job-application history.

Visit nejmjobs.org for more information. 